\title{
Catalytic Synthesis of $\gamma$-Lactams via Direct Annulations of Enals and N-Sulfonylimines
}

\author{
Ming He and Jeffrey W. Bode* \\ Department of Chemistry and Biochemistry, University of California, \\ Santa Barbara, CA 93106-9510
}

\section{Supporting Information}

\section{Table of Contents:}

I. General Methods 2

II. Experimental Procedures and Characterization Data 2

III. Determination of Relative Stereochemistry 12

IV. Deuterium-labelling Studies 12

V. NMR Studies of Electrophilic Inhibition 13

VI. Spectral Data of Selected Lactams 14 
General Methods. All reactions utilizing air- or moisture-sensitive reagents were performed in dried glassware under an atmosphere of dry Ar. ${ }^{\mathrm{i} r O H}$, and tert-BuOH were distilled from Na. $\mathrm{CH}_{2} \mathrm{Cl}_{2}$ was distilled over $\mathrm{CaH}_{2}$. THF, $\mathrm{CH}_{3} \mathrm{CN}$ and EtOAc were dried by passage over activated alumina under Ar atmosphere. Imidazolium salts were prepared according to a reported protocol. ${ }^{1}$ All aldehydes were purified by distillation prior to use. DBU was distilled from $\mathrm{KOH}$. Other reagents were used without further purification. Chemical yields of the lactam products were reported for the combined yields of the diastereomers. Thin layer chromatography (TLC) was performed on Merck precoated plates (silica gel $60 \mathrm{~F}_{254}$, Art 5715, $0.25 \mathrm{~mm}$ ) and were visualized by fluorescence quenching under UV light or by staining with phosphomolybdic acid. Silica-gel preparative thin-layer chromatography (PTLC) was performed using plates prepared from Merck Kieselgel $60 \mathrm{PF}_{254}$ (Art 7747). Column chromatography was performed on E. Merck Silica Gel 60 (230-400 Mesh) using a forced flow of 0.5-1.0 bar. ${ }^{1} \mathrm{H}$ NMR (400 $\mathrm{MHz})$ and ${ }^{13} \mathrm{C}$ NMR (100 MHz) were measured on a Varian Unity 400 spectrometer. The peaks with * indicate peaks of the minor diastereomer (trans-). Chemical shifts are expressed in parts per million (PPM) downfield from residual solvent peaks and coupling constants are reported as Hertz $(\mathrm{Hz})$. Splitting patterns are indicated as follows: br, broad; s, singlet; d, doublet; t, triplet; q, quartet; m, multiplet. Infrared (IR) spectra were recorded on a JASCO FT/IR-430 spectrophotometer and are reported as wavenumber $\left(\mathrm{cm}^{-1}\right)$. Compounds that are not numbered in the manuscript are labelled as $\mathbf{S 1}, \mathbf{S 2}$, etc.

\section{General Procedure for Catalytic Annulations of Enals and Imines.}

The reaction of enal $\mathbf{2}$ and imine $\mathbf{7}$ is representative. Into an oven dried $20 \mathrm{~mL}$ vial was weighed the enal 2 ( $0.018 \mathrm{~g}, 0.10 \mathrm{mmol}, 1.0$ equiv), imine 7 (0.029 g, $0.10 \mathrm{mmol}, 1.0$ equiv) and IMes-Cl (5.2mg, $0.015 \mathrm{mmol}, 0.15$ equiv). The vial was closed with crimp seal, evacuated, and back-filled with argon. To this mixture was added $1.0 \mathrm{~mL}$ tert$\mathrm{BuOH}$, followed by $\mathrm{DBU}(1.5 \mu \mathrm{L}, 0.01 \mathrm{mmol}, 0.10$ equiv). The resulting solution was stirred $14 \mathrm{~h}$ at $60{ }^{\circ} \mathrm{C}$. The reaction mixture was concentrated under reduced pressure, and

(1) (a) Arduengo, A. J., III U.S. Patent 5077 414, 1991. (b) We are grateful to Prof. Steven Nolan (U. New Orleans) for suggested improvements to this protocol. 
then the residue was purified by flash chromatography (4:1 hexane/EtOAc) to afford the lactam products as a white solid (0.036 g, 75\% yield).

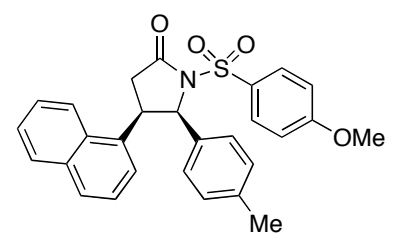

cis-1-(4-methoxyphenylsulfonyl)-4-(naphthalen-1-yl)-5-p-tolylpyrrolidin-2-one (S1)

Prepared according to the general procedure in $75 \%$ as a $10: 1$ mixture of lactam diastereomers. (0.10 mmol scale)

${ }^{1}$ H NMR $\left(400 \mathrm{MHz}, \mathrm{CDCl}_{3}\right) \delta 8.26(\mathrm{~d}, 1 \mathrm{H}, J=8.6), 7.85(\mathrm{~d}, 1 \mathrm{H}, J=7.1), 7.74(\mathrm{~d}, 2 \mathrm{H}, J$ $=9.1), 7.68-7.75(\mathrm{~m}, 1 \mathrm{H}), 7.55-7.63(\mathrm{~m}, 2 \mathrm{H}), 7.04-7.08(\mathrm{~m}, 1 \mathrm{H}), 6.87-6.89(\mathrm{~m}$, $2 \mathrm{H}), 6.61-6.65(\mathrm{~m}, 3 \mathrm{H}), 6.24(\mathrm{~d}, 2 \mathrm{H}, J=8.0), 5.97(\mathrm{~d}, 1 \mathrm{H}, J=7.7), 4.82-4.89(\mathrm{~m}, 1 \mathrm{H})$, 3.89 (s, 3H), 3.31 (dd, 1H, $J=13.5,7.4), 2.72(\mathrm{dd}, 1 \mathrm{H}, J=17.0,7.4), 2.13(\mathrm{~s}, 3 \mathrm{H})$

${ }^{13}$ C NMR $\left(100 \mathrm{MHz}, \mathrm{CDCl}_{3}\right) \delta 172.8,164.2,137.6,133.8,132.1,131.9,131.4,131.0$, $129.8,129.4,128.7,128.2,127.0,126.3,126.0,124.9,124.3,122.9,113.8,66.2,55.9$, 41.0, 34.6, 21.1

IR (thin film) $v$ 3055, 2987, 2306, 1736, 1596, 1422, 1266, 1166, 896, $740 \mathrm{~cm}^{-1}$

ESI-MS: $471.0 \quad\left(\mathrm{M}^{+}\right)$

MP: $200-202^{\circ} \mathrm{C}$

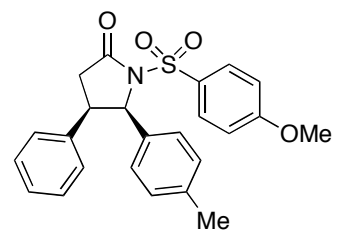

cis-1-(4-methoxyphenylsulfonyl)-4-phenyl-5-p-tolylpyrrolidin-2-one (8)

Prepared according to the general procedure in $70 \%$ as a $4: 1$ mixture of lactam diastereomers. (0.10 mmol scale)

${ }^{1} \mathbf{H}$ NMR $\left(400 \mathrm{MHz}, \mathrm{CDCl}_{3}\right) \delta 7.71(\mathrm{~d}, 2 \mathrm{H}, J=9.0), 7.06-7.17(\mathrm{~m}, 4 \mathrm{H}), 6.81-6.88(\mathrm{~m}$, $3 \mathrm{H}), 6.76-6.78(\mathrm{~m}, 2 \mathrm{H}), 6.53(\mathrm{~d}, 2 \mathrm{H}, J=8.1), 5.57(\mathrm{~d}, 1 \mathrm{H}, J=8.0), 5.26^{*}(\mathrm{~d}, 1 \mathrm{H}, J=$ 2.3), $4.06(\mathrm{~m}, 1 \mathrm{H}), 3.34^{*}(\mathrm{~m}, 1 \mathrm{H}), 3.87(\mathrm{~s}, 3 \mathrm{H}), 3.88^{*}(\mathrm{~s}, 3 \mathrm{H}), 3.03((\mathrm{dd}, 1 \mathrm{H}, J=17.2$, 13.7), 3.10* (dd, $1 \mathrm{H}, J=17.8,8.8), 2.68$ (dd, $1 \mathrm{H}, J=17.2,7.8), 2.60 *$ (dd, $1 \mathrm{H}, J=17.8$, 3.6), $2.23(\mathrm{~s}, 3 \mathrm{H}), 2.37 *(\mathrm{~s}, 3 \mathrm{H})$

${ }^{13}$ C NMR $\left(100 \mathrm{MHz}, \mathrm{CDCl}_{3}\right) \delta 172.8,164.1,142.6,138.2,137.8,135.6,132.3,131.3$, $131.1,129.9,129.8,129.4,128.9,128.4,128.2$, 127.8, 127.6, 127.2, 126.4, 125.9, 113.9, $113.8,71.1,67.5,60.6,55.9,47.0,45.0,37.9,35.3,21.2,14.4$.

IR (thin film) $v$ 3059, 2982, 2304, 1735, 1596, 1497, 1363, 1266, 1165, 1092, 722, 703 $\mathrm{cm}^{-1}$

ESI-MS: $421.0 \quad\left(\mathrm{M}^{+}\right)$

MP: $158-161^{\circ} \mathrm{C}$ 


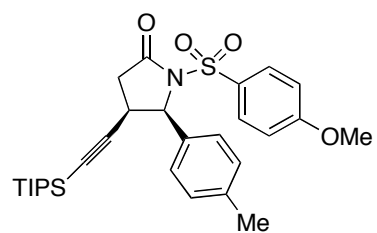

cis-1-(4-methoxyphenylsulfonyl)-5-p-tolyl-4-(2-(triisopropylsilyl)ethynyl)pyrrolidin2-one (24)

Into an oven dried $20 \mathrm{~mL}$ vial was weighed the imine (98 $\mathrm{mg}, 0.11 \mathrm{mmol}, 1.0$ equiv), IMes-Cl (5.2 mg, 0.015 mmol, 0.15 equiv), DBU (1.7 $\mu \mathrm{L}, 0.011 \mathrm{mmol}, 0.10$ equiv) and $0.50 \mathrm{ml}$ tert-BuOH . The vial was closed with crimp seal, evacuated, and back-filled with argon. To this mixture was slowly injected $0.80 \mathrm{~mL}$ tert-BuOH solution of the enal (27 $\mathrm{mg}, 0.11 \mathrm{mmol}, 1.0$ equiv.) over $3 \mathrm{~h}$ at $40^{\circ} \mathrm{C}$. The resulting mixture was stirred $12 \mathrm{~h}$ at 40 ${ }^{\circ} \mathrm{C}$. The reaction mixture was concentrated under reduced pressure and the residue was purified by flash chromatography $(5: 1$ hexane/EtOAc) to afford the lactam products in $51 \%$ as a $10: 1$ mixture of diastereomers.

${ }^{1}$ H NMR $\left(400 \mathrm{MHz}, \mathrm{CDCl}_{3}\right) \delta 7.56(\mathrm{~d}, 2 \mathrm{H}, J=8.9), 7.81 *(\mathrm{~d}, 2 \mathrm{H}, J=8.9), 7.01(\mathrm{~d}, 2 \mathrm{H}, J$ $=8.0), 6.89(\mathrm{~d}, 2 \mathrm{H}, J=8.0), 6.79(\mathrm{~d}, 2 \mathrm{H}, J=8.9), 5.45(\mathrm{~d}, 1 \mathrm{H}, J=8.1), 5.35^{*}(\mathrm{~d}, 1 \mathrm{H}, J=$ 2.6), $3.84(\mathrm{~s}, 3 \mathrm{H}), 3.86^{*}(\mathrm{~s}, 3 \mathrm{H}), 3.70(\mathrm{~m}, 1 \mathrm{H}), 3.01^{*}(\mathrm{~m}, 1 \mathrm{H}), 2.83(\mathrm{dd}, 1 \mathrm{H}, J=17.7$, 9.1), 2.91* (dd, $1 \mathrm{H}, J=17.2,8.4$ ), 2.76 (dd, $1 \mathrm{H}, J=17.7,12.1), 2.52 *$ (dd, $1 \mathrm{H}, J=17.1$, $3.4), 2.30$ (s, 3H), 2.36* (s, 3H), $0.81-0.84(\mathrm{~m} \mathrm{21H})$

${ }^{13} \mathrm{C}$ NMR $\left(100 \mathrm{MHz}, \mathrm{CDCl}_{3}\right) \delta 171.4,164.1,138.4,133.1,131.2,131.1,129.8,129.6$, 129.2 , 127.6, 125.8, 114.0, 113.8, 103.1, 87.5, 65.3, 55.9, 37.8, 34.9, 32.0, 21.3, 18.8, $18.5,11.2,11.1$

IR (thin film) $v$ 3054, 2986, 2305, 1734, 1641, 1596, 1497, 1421, 1364, 1262, 1166, 1092, 896, 836, $701 \mathrm{~cm}^{-1}$

ESI-MS: $\quad 526.2 \quad(\mathrm{M}+\mathrm{H})$

MP: $113-115^{\circ} \mathrm{C}$ 


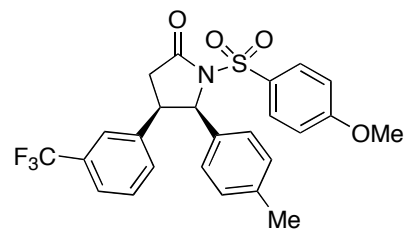

cis-1-(4-methoxyphenylsulfonyl)-5-p-tolyl-4-(3-(trifluoromethyl)phenyl)pyrrolidin-2one (18)

Prepared according to the general procedure in $70 \%$ as a $3: 2$ mixture of lactam diastereomers. (0.10 mmol scale)

${ }^{1} \mathbf{H}$ NMR (400 MHz, $\left.\mathrm{CDCl}_{3}\right) \delta 7.73(\mathrm{~d}, 2 \mathrm{H}, J=8.9), 7.31-7.42(\mathrm{~m}, 1 \mathrm{H}), 7.16-7.24(\mathrm{~m}$, $2 \mathrm{H}), 6.99(\mathrm{~d}, 1 \mathrm{H}, J=7.7), 6.84-6.90(\mathrm{~m}, 4 \mathrm{H}), 6.49(\mathrm{~d}, 2 \mathrm{H}, J=8.9), 5.58(\mathrm{~d}, 1 \mathrm{H}, J=7.8)$, $5.25^{*}(\mathrm{~d}, 1 \mathrm{H}, J=2.5), 4.14(\mathrm{~m}, 1 \mathrm{H}), 3.41^{*}(\mathrm{~m}, 1 \mathrm{H}), 3.87(\mathrm{~s}, 3 \mathrm{H}), 3.00 \quad(\mathrm{dd}, 1 \mathrm{H}, J=$ $17.2,13.5), 3.15^{*}(\mathrm{dd}, 1 \mathrm{H}, J=17.8,8.9), 2.72$ (dd, $\left.1 \mathrm{H}, J=17.2,8.0\right), 2.59^{*}(\mathrm{dd}, 1 \mathrm{H}$, $J=17.8,3.2), 2.23(\mathrm{~s}, 3 \mathrm{H}), 2.38^{*}(\mathrm{~s}, 3 \mathrm{H})$.

${ }^{13}$ C NMR $\left(100 \mathrm{MHz}, \mathrm{CDCl}_{3}\right) \delta 172.6,172.1,164.3,164.2,143.4,138.5,137.0,136.9$, $131.8,131.4,131.0,129.9$, 129.8, 129.6, 129.1, 128.9, 127.0, 125.9, 125.0, 124.8, 124.4, $123.5,114.0,113.9,70.6,67.2,55.9,46.9,44.7,37.8,35.5,21.3,21.1$

IR (thin film) $v$ 3055, 2987, 2305, 1738, 1596, 1498, 1330, 1266, 1164, 1128, 896, 835, $805,744 \mathrm{~cm}^{-1}$

ESI-MS: $490.1 \quad(\mathrm{M}+\mathrm{H})$

MP: $119-125^{\circ} \mathrm{C}$

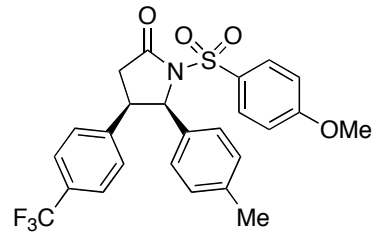

cis-1-(4-methoxyphenylsulfonyl)-5-p-tolyl-4-(4-(trifluoromethyl)phenyl)pyrrolidin-2one (20)

Prepared according to the general procedure in $70 \%$ combined yield (cis : trans $=3.5: 1$ ).

The diastereomers were separated by PTLC (4:1 hexane/EtOAc). (0.10 mmol scale)

${ }^{1} \mathbf{H}$ NMR $\left(400 \mathrm{MHz}, \mathrm{CDCl}_{3}\right) \delta 7.66(\mathrm{~d}, 2 \mathrm{H}, J=8.5), 7.31(\mathrm{~d}, 2 \mathrm{H}, J=8.3), 6.80-6.86(\mathrm{~m}$, $6 \mathrm{H}), 6.49(\mathrm{~d}, 2 \mathrm{H}, J=8.5), 5.57(\mathrm{~d}, 1 \mathrm{H}, J=7.8), 4.07-4.11(\mathrm{~m}, 1 \mathrm{H}), 3.83(\mathrm{~s}, 3 \mathrm{H}), 2.99(\mathrm{dd}$, $1 \mathrm{H}, J=17.2,13.5), 2.68(\mathrm{dd}, 1 \mathrm{H}, J=17.2,8.0), 2.21(\mathrm{~s}, 3 \mathrm{H})$

${ }^{13} \mathrm{C}$ NMR $\left(100 \mathrm{MHz}, \mathrm{CDCl}_{3}\right) \delta 172.1,164.2,139.9,138.3,131.9,131.3,129.8,129.2$, $128.7,127.1,125.3,125.2,113.9,67.1,55.9,44.7,44.7,35.4,21.3$

IR (thin film) $v$ 3055, 2987, 2305, 1738, 1596, 1498, 1362, 1328, 1261, 1162, 1124, 896, $836,749,674 \mathrm{~cm}^{-1}$

ESI-MS: $490.1 \quad(\mathrm{M}+\mathrm{H})$

MP: $164-166^{\circ} \mathrm{C}$ 


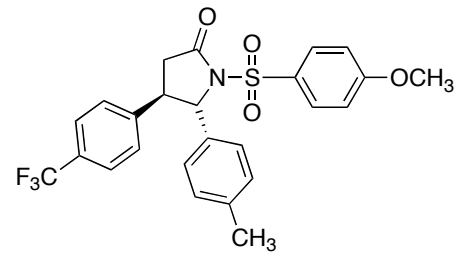

trans-1-(4-methoxyphenylsulfonyl)-5-p-tolyl-4-(4-(trifluoromethyl)phenyl)pyrrolidin -2-one (S2)

${ }^{1} \mathbf{H}$ NMR $\left(400 \mathrm{MHz}, \mathrm{CDCl}_{3}\right) \delta 7.67(\mathrm{~d}, 2 \mathrm{H}, J=9.1), 7.55(\mathrm{~d}, 2 \mathrm{H}, J=8.0), 7.16-7.21(\mathrm{~m}$, $4 \mathrm{H}), 7.10(\mathrm{~d}, 2 \mathrm{H}, J=8.0), 6.87(\mathrm{~d}, 2 \mathrm{H}, J=9.0), 5.57(\mathrm{~d}, 1 \mathrm{H}, J=7.8), 4.07-4.11(\mathrm{~m}, 1 \mathrm{H})$, $3.83(\mathrm{~s}, 3 \mathrm{H}), 2.99(\mathrm{dd}, 1 \mathrm{H}, J=17.2,13.5), 2.68(\mathrm{dd}, 1 \mathrm{H}, J=17.2,8.0), 2.21(\mathrm{~s}, 3 \mathrm{H})$

${ }^{13}$ C NMR (100 MHz, $\left.\mathrm{CDCl}_{3}\right) \delta$ 172.6, 164.3, 138.6, 136.9, 131.0, 129.9, 129.7, 129.1, $127.3,126.9,126.4,126.3,125.9,113.9,70.6,58.1,46.8,37.7,21.3$

IR (thin film) $v$ 3054, 2987, 2395, 1735, 1596, 1497, 1421, 1364, 1266, 1165, 896, 739, $705 \mathrm{~cm}^{-1}$

ESI-MS: $490.1(\mathrm{M}+\mathrm{H})$

MP: $182-183^{\circ}$

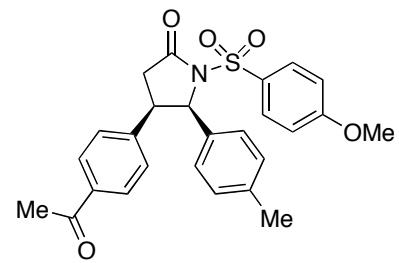

cis-4-(4-acetylphenyl)-1-(4-methoxyphenylsulfonyl)-5-p-tolylpyrrolidin-2-one (22)

Prepared according to the general procedure in $65 \%$ as a $3.5: 1$ mixture of lactam diastereomers. (0.10 $\mathrm{mmol} \mathrm{scale})$

${ }^{1} \mathbf{H}$ NMR $\left(400 \mathrm{MHz}, \mathrm{CDCl}_{3}\right) \delta$ 7.89-7.65 (m, 4H), 7.19-6.97(m, 2H), 6.89-6.81(m, $4 \mathrm{H}), 6.53(\mathrm{~d}, 2 \mathrm{H}, J=7.8), 5.61(\mathrm{~d}, 1 \mathrm{H}, J=7.8), 5.23^{*}(\mathrm{~d}, 1 \mathrm{H}, J=2.5), 4.12(\mathrm{~m}, 1 \mathrm{H})$, $3.40^{*}(\mathrm{~m}, 1 \mathrm{H}), 3.86(\mathrm{~s}, 3 \mathrm{H}), 3.89^{*}(\mathrm{~s}, 3 \mathrm{H}), 3.06(\mathrm{dd}, 1 \mathrm{H}, J=17.2,13.5), 3.12 *(\mathrm{dd}, 1 \mathrm{H}, J$ $=17.8,8.8), 2.72(\mathrm{dd}, 1 \mathrm{H}, J=17.2,7.8), 2.61 *(\mathrm{dd}, 1 \mathrm{H}, J=17.8,4.2), 2.51(\mathrm{~s}, 3 \mathrm{H}), 2.61 *$ $(\mathrm{s}, 3 \mathrm{H}), 2.22(\mathrm{~s}, 3 \mathrm{H}), 2.37 *(\mathrm{~s}, 3 \mathrm{H})$

${ }^{13}$ C NMR $\left(100 \mathrm{MHz}, \mathrm{CDCl}_{3}\right) \delta 192.8,172.2,164.1,141.9,138.2,136.2,131.9,131.3$, $131.0,129.9,129.7,129.5,129.1,128.8,128.5,128.4,127.2,126.8,125.9,114.4,113.9$, $113.9,70.6,67.2,55.9,47.0,44.8,37.7,35.3,26.8,21.3$

IR (thin film) $v$ 3054, 2986, 2305, 1734, 1641, 1596, 1497, 1421, 1364, 1262, 1166, 1092, 896, 836, $701 \mathrm{~cm}^{-1}$

ESI-MS: $\quad 464.1 \quad(\mathrm{M}+\mathrm{H})$

MP: $172-176^{\circ} \mathrm{C}$ 


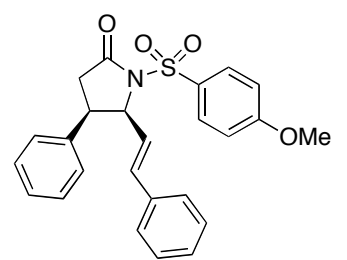

cis-1-(4-methoxyphenylsulfonyl)-4-phenyl-5-(2-p-tolylethylidene)pyrrolidin-2-one (14)

Into an oven dried flask was weighed enal 6 ( $0.90 \mathrm{~g}, 6.8 \mathrm{mmol}, 1.0$ equiv), imine 13 (2.05 $\mathrm{g}, 6.8 \mathrm{mmol}, 1.0$ equiv) and IMes- $\mathrm{Cl}(0.35 \mathrm{~g}, 1.0 \mathrm{mmol}, 0.15$ equiv). The flask was evacuated, and back-filled with argon. To this mixture was added $68 \mathrm{~mL}$ tert-BuOH , followed by the addition of $\mathrm{DBU}(0.10 \mathrm{~mL}, 0.68 \mathrm{mmol}, 0.10$ equiv). The resulting solution was stirred $14 \mathrm{~h}$ at room temperature. The reaction mixture was concentrated under reduced pressure, and then residue purified by flash chromatography $(4: 1$ hexane/EtOAc) to afford the products as an 8:1 mixture of lactam diastereomers $(1.8 \mathrm{~g}$, $4.2 \mathrm{mmol}, 61 \%$ yield).

${ }^{1} \mathbf{H}$ NMR (400 MHz, $\left.\mathrm{CDCl}_{3}\right) \delta 7.96(\mathrm{~d}, 2 \mathrm{H}, J=8.9), 7.28-7.21(\mathrm{~m}, 3 \mathrm{H}), 7.15-7.04(\mathrm{~m}$, $2 \mathrm{H}), 6.86(\mathrm{~d}, 2 \mathrm{H}, J=8.1), 6.39(\mathrm{~d}, 1 \mathrm{H}, J=15.7), 5.58(\mathrm{dd}, 1 \mathrm{H}, J=15.7,8.2), 5.31(\mathrm{~m}$, $1 \mathrm{H}), 4.93 *(\mathrm{~m}, 1 \mathrm{H}), 3.99(\mathrm{~m}, 1 \mathrm{H}), 3.34^{*}(\mathrm{~m}, 1 \mathrm{H}), 3.83(\mathrm{~s}, 3 \mathrm{H}), 3.86^{*}(\mathrm{~s}, 3 \mathrm{H}), 2.98$ (dd, $1 \mathrm{H}, J=17.2,13.2), 3.15^{*}(\mathrm{dd}, 1 \mathrm{H}, J=17.6,8.4), 2.72(\mathrm{dd}, 1 \mathrm{H}, J=17.2,7.2), 2.61^{*}(\mathrm{dd}$, $1 \mathrm{H}, J=17.6,3.2), 2.51(\mathrm{~s}, 3 \mathrm{H}), 2.61 *(\mathrm{~s}, 3 \mathrm{H}), 2.22(\mathrm{~s}, 3 \mathrm{H}), 2.37 *(\mathrm{~s}, 3 \mathrm{H})$

${ }^{13}$ C NMR (100 MHz, $\left.\mathrm{CDCl}_{3}\right) \delta 172.1,164.1,135.8,135.7,134.7,131.3,131.1,130.3$, $129.4,128.9,128.7,128.1,127.9,126.9,126.7,126.6,123.2,114.1,65.8,55.8,43.5,35.2$ IR (thin film) $v$ 3054, 2987, 2305, 1735, 1654, 1596, 1498, 1421, 1265, 1166, 1092, 898, $835,739 \mathrm{~cm}^{-1}$

ESI-MS: $\quad 434.1 \quad(\mathrm{M}+\mathrm{H})$

MP: $135-136^{\circ} \mathrm{C}$

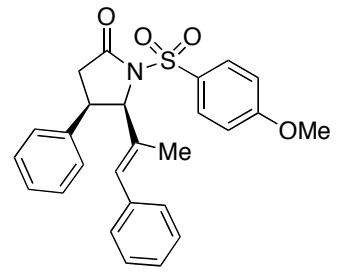

cis-1-(4-methoxyphenylsulfonyl)-4-phenyl-5-(2-p-tolylprop-1-enyl)pyrrolidin-2-one (16)

Prepared according to the general procedure in $62 \%$ as a 5:1 mixture of lactam diastereomers.

${ }^{1} \mathbf{H}$ NMR $\left(400 \mathrm{MHz}, \mathrm{CDCl}_{3}\right) \delta 8.00(\mathrm{~m}, 2 \mathrm{H}), 7.93 *(\mathrm{~m}, 2 \mathrm{H}), 7.35-7.20(\mathrm{~m}, 8 \mathrm{H}), 6.93-$ $6.85(\mathrm{~m}, 4 \mathrm{H}), 6.25(\mathrm{~s}, 1 \mathrm{H}), 6.45^{*}(\mathrm{~s}, 1 \mathrm{H}), 5.19(\mathrm{~d}, 1 \mathrm{H}, J=9.3), 4.78^{*}(\mathrm{~d}, 1 \mathrm{H}, J=1.1)$, 
$4.05(\mathrm{~m}, 1 \mathrm{H}), 3.36 *(\mathrm{~m}, 1 \mathrm{H}), 3.84(\mathrm{~s}, 3 \mathrm{H}), 3.87 *$ (s, 3H), 3.07 (dd, $1 \mathrm{H}, J=17.4,13.5)$, $3.27 *$ (dd, $1 \mathrm{H}, J=18.0,8.6), 2.74$ (dd, $1 \mathrm{H}, J=17.4,8.3), 2.59 *$ (dd, $1 \mathrm{H}, J=18.0,2.6$ ), 1.09 (d, 3H, $J=1.4), 1.86 *(\mathrm{~d}, 3 \mathrm{H}, J=1.2$ ),

${ }^{13}$ C NMR $\left(100 \mathrm{MHz}, \mathrm{CDCl}_{3}\right) \delta 173.3,172.6,164.1,143.1,136.5,135.7,135.0,133.4$, $131.2,131.1,130.1,130.0,129.4,129.3,129.1,128.7,128.4,128.3,128.3,127.9,127.8$, 127.7, 127.2, 127.1, 126.3, 114.1, 74.6, 70.1, 55.9, 43.0, 42.8, 38.4, 35.4, 16.7, 15.0

IR (thin film) v 3067, 3024, 2957, 2253, 1731, 1596, 1498, 1364, 1264, 1165, 1092, $1029,834,741,650 \mathrm{~cm}^{-1}$

ESI-MS: $\quad 448.1 \quad(\mathrm{M}+\mathrm{H})$

MP: $148-151^{\circ} \mathrm{C}$

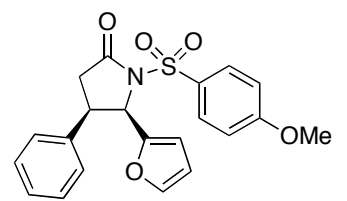

cis-5-(furan-2-yl)-1-(4-methoxyphenylsulfonyl)-4-phenylpyrrolidin-2-one (12)

Prepared according to the general procedure with 2.0 equiv enal $\mathbf{6}$ and 1.0 equiv imine $\mathbf{1 1}$ at $75^{\circ} \mathrm{C}$ for $63 \mathrm{~h}$ in $73 \%$ as a $1.7: 1$ mixture of lactam diastereomers.

${ }^{1} \mathbf{H}$ NMR $\left(400 \mathrm{MHz}, \mathrm{CDCl}_{3}\right) \delta$ 7.60-7.52 (m, 2H), 7.35-7.05 (m, 3H), 7.15-7.04 (m, $4 \mathrm{H}), 6.95-6.92(\mathrm{~m}, 1 \mathrm{H}), 6.88-6.81(\mathrm{~m}, 3 \mathrm{H}), 6.14-6.09(\mathrm{~m}, 1 \mathrm{H}), 6.44-6.39 *(\mathrm{~m}, 1 \mathrm{H})$, $5.59(\mathrm{~d}, 1 \mathrm{H}, J=7.7), 5.33^{*}(\mathrm{~d}, 1 \mathrm{H}, J=1.7), 4.03(\mathrm{~m}, 1 \mathrm{H}), 3.85^{*}(\mathrm{~m}, 1 \mathrm{H}), 3.84(\mathrm{~s}, 3 \mathrm{H})$, $3.86^{*}(\mathrm{~s}, 3 \mathrm{H}), 3.25$ (dd, $\left.1 \mathrm{H}, J=17.0,13.4\right), 3.27 *(\mathrm{dd}, 1 \mathrm{H}, J=17.5,9.0), 2.76$ (dd, $1 \mathrm{H}, J$ $=17.0,8.2), 2.63 *(\mathrm{dd}, 1 \mathrm{H}, J=17.5,2.2)$

${ }^{13}$ C NMR $\left(100 \mathrm{MHz}, \mathrm{CDCl}_{3}\right) \delta 172.4,163.9,149.3,143.0,142.5,135.6,134.7,131.3$, $130.8,129.5,129.4,128.9,128.7,128.6,128.4,128.1,127.9,127.8,127.6,126.8,126.4$, $123.2,114.1,114.0,113.9,111.3,110.7,110.5,94.6,63.6,61.1,55.8,43.8,43.6,43.1$, $38.7,36.1$

IR (thin film) v 3054, 2987, 2305, 1735, 1596, 1498, 1421, 1267, 1166, 896, $739 \mathrm{~cm}^{-1}$

ESI-MS: $398.1 \quad(\mathrm{M}+\mathrm{H})$

MP: $118-121^{\circ} \mathrm{C}$

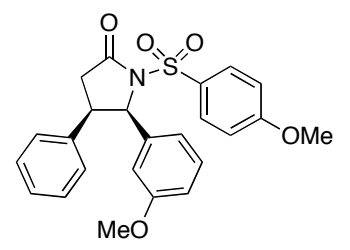

cis-5-(3-methoxyphenyl)-1-(4-methoxyphenylsulfonyl)-4-phenylpyrrolidin-2-one (10)

Prepared according to the general procedure in $69 \%$ as a $3: 1$ mixture of lactam diastereomers.

${ }^{1} \mathbf{H}$ NMR $\left(400 \mathrm{MHz}, \mathrm{CDCl}_{3}\right) \delta$ 7.76-7.69 (m, 2H,), 7.32-7.24 (m, 2H), 7.14-7.05(m, $3 \mathrm{H}), 6.96-6.84(\mathrm{~m}, 3 \mathrm{H}), 6.82-6.75(\mathrm{~m}, 1 \mathrm{H}), 6.23(\mathrm{~m}, 1 \mathrm{H}), 6.65^{*}(\mathrm{~m}, 1 \mathrm{H}), 6.05(\mathrm{~m}$, $1 \mathrm{H}), 6.23^{*}(\mathrm{~m}, 1 \mathrm{H}), 5.56(\mathrm{~d}, 1 \mathrm{H}, J=7.8), 5.26^{*}(\mathrm{~d}, 1 \mathrm{H}, J=2.3), 4.10(\mathrm{~m}, 1 \mathrm{H}), 3.36^{*}(\mathrm{~m}$, 
$1 \mathrm{H}), 3.87(\mathrm{~s}, 3 \mathrm{H}), 3.88^{*}(\mathrm{~s}, 3 \mathrm{H}), 3.48(\mathrm{~s}, 3 \mathrm{H}), 3.75^{*}(\mathrm{~s}, 3 \mathrm{H}), 3.04(\mathrm{dd}, 1 \mathrm{H}, J=17.2,14.0)$, $3.10 *(\mathrm{dd}, 1 \mathrm{H}, J=18.0,9.2), 2.71(\mathrm{dd}, 1 \mathrm{H}, J=17.2,7.6), 2.60 *(\mathrm{dd}, 1 \mathrm{H}, J=18.0,3.2)$

${ }^{13} \mathrm{C}$ NMR $\left(100 \mathrm{MHz}, \mathrm{CDCl}_{3}\right) \delta 173.2,172.7,164.2,160.2,159.4,142.6,141.9,136.7$, $135.5,131.3,131.1,130.3,129.9,129.4,129.2$, 128.4, 128.2, 127.8, 127.7, 126.4, 119.5, $118.2,114.2,113.9,112.5,111.5,67.6,55.9,55.4,55.1,46.8,45.0,38.0,35.2$

IR (thin film) $v$ 3054, 2987, 2305, 1735, 1685, 1422, 1266, 1165, 896, $742 \mathrm{~cm}^{-1}$

ESI-MS: $438.1 \quad(\mathrm{M}+\mathrm{H})$

MP: $198-202^{\circ} \mathrm{C}$

\section{Preparation of Enals}

The enals 17, 19 and 21 were prepared according to the procedure of Battistuzzi et. al. ${ }^{2}$

To a stirred solution of the aryl halide (1.0 equiv) in DMF was added acrolein diethyl acetal (3.0 equiv), $\mathrm{n}-\mathrm{Bu}_{4} \mathrm{NOAc}$ (2.0 equiv), $\mathrm{K}_{2} \mathrm{CO}_{3}$ (1.5 equiv), $\mathrm{KCl}$ (1.0 equiv), and $\mathrm{Pd}(\mathrm{OAc})_{2}(3.0 \mathrm{~mol} \%)$. The mixture was stirred for $1.5 \mathrm{~h}$ at $90{ }^{\circ} \mathrm{C}$. After cooling, $2 \mathrm{~N} \mathrm{HCl}$ was slowly added and the reaction mixture was stirred at room temperature for $10 \mathrm{~min}$. Then, it was diluted with ether and washed with water. The organic layer was dried over $\mathrm{Na}_{2} \mathrm{SO}_{4}$ and concentrated under reduced pressure. The residue was purified by chromatography to give the products. The spectral data were compared to previous reports. $^{2}$

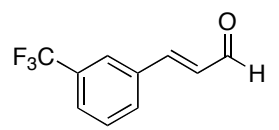

(E)-3-(3-(trifluoromethyl)phenyl)acrylaldehyde (17)

${ }^{1} \mathbf{H}$ NMR $\left(400 \mathrm{MHz}, \mathrm{CDCl}_{3}\right) \delta 9.75(\mathrm{~d}, 1 \mathrm{H}, J=7.5), 7.81-7.57(\mathrm{~m}, 4 \mathrm{H}), 7.51(\mathrm{~d}, 1 \mathrm{H}, J=$ $16.1), 6.78(\mathrm{dd}, 1 \mathrm{H}, J=16.1,7.5)$

${ }^{13}$ C NMR $\left(100 \mathrm{MHz}, \mathrm{CDCl}_{3}\right) \delta 193.4,150.6,134.9,131.4,130.2,129.9,127.8,127.7$, $125.4,125.3$

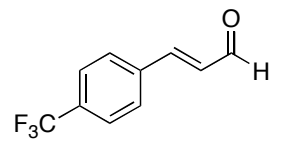

(E)-3-(4-(trifluoromethyl)phenyl)acrylaldehyde (19)

${ }^{1} \mathbf{H}$ NMR (400 MHz, $\left.\mathrm{CDCl}_{3}\right) \delta 9.75(\mathrm{~d}, 1 \mathrm{H}, J=7.5), 7.69(\mathrm{~s}, 4 \mathrm{H}), 7.51$ (d, 1H, $\left.J=16.1\right)$, $6.77(\mathrm{dd}, 1 \mathrm{H}, J=16.1,7.5)$

${ }^{13} \mathbf{C}$ NMR $\left(100 \mathrm{MHz}, \mathrm{CDCl}_{3}\right) \delta 193.4,150.5,130.7,128.8,126.3,126.2$,

(2) Battistuzzi, G.; Cacchi, S.; Fabrizi, G.; Org. Lett. 2003, 5, 777-780 


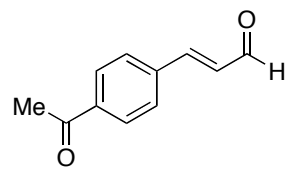

(E)-3-(4-acetylphenyl)acrylaldehyde (21)

${ }^{1} \mathbf{H}$ NMR (400 MHz, $\left.\mathrm{CDCl}_{3}\right) \delta 9.74(\mathrm{~d}, 1 \mathrm{H}, J=7.5), 8.00(\mathrm{~d}, 2 \mathrm{H}, J=8.3), 7.66(\mathrm{~d}, 1 \mathrm{H}, J$ $=8.3), 7.51(\mathrm{~d}, 1 \mathrm{H}, J=16.1), 6.77(\mathrm{dd}, 1 \mathrm{H}, J=16.1,7.5)$

${ }^{13}$ C NMR $\left(100 \mathrm{MHz}, \mathrm{CDCl}_{3}\right) \delta 197.5,193.6,151.0,138.8,138.3,130.6,129.2,128.8$, 26.9

\section{Preparation of Imines}

The imines 7, 9, 13 and 15 were prepared according to the following procedure.

The corresponding dimethyl acetal (1.0 eqiv) and the arylsulfonamide (1.0 eqiv) were mixed in a flask equipped with a Dean-Stark condenser. The neat mixture was heated to $180{ }^{\circ} \mathrm{C}$ for $30 \mathrm{~min}$. The resulting melt was cooled to room temperature and the solid was crystallized from toluene to yield the product.

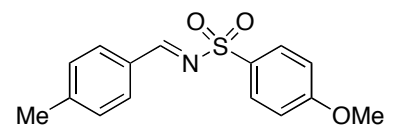

(E)-4-methoxy-N-((E)-p-toylethylidene)benzenesulfonamide (7)

${ }^{1} \mathbf{H}$ NMR (400 MHz, $\left.\mathrm{CDCl}_{3}\right) \delta 8.96(\mathrm{~s}, 1 \mathrm{H}), 7.93$ (d, 2H, $\left.J=8.7\right), 7.81$ (d, 2H, $J=8.0$ ), $7.29(\mathrm{~d}, 2 \mathrm{H}, J=8.0), 7.01(\mathrm{~d}, 2 \mathrm{H}, J=8.7), 3.88(\mathrm{~s}, 3 \mathrm{H}), 2.43(\mathrm{~s}, 3 \mathrm{H})$

${ }^{13}$ C NMR $\left(100 \mathrm{MHz}, \mathrm{CDCl}_{3}\right) \delta 169.7,163.8,146.5,131.6,130.4,130.1,130.0,129.9$, $114.6,55.9,22.2$

IR (thin film) $v$ 3054, 2987, 2305, 1597, 1421, 1265, 1154, 1092, 896, 809, $740 \mathrm{~cm}^{-1}$

ESI-MS: $290.1 \quad(\mathrm{M}+\mathrm{H})$

MP: $106-108^{\circ} \mathrm{C}$

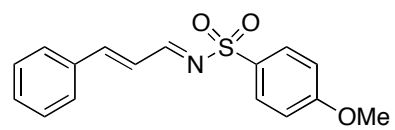

(E)-4-methoxy-N-((E)-3-phenylallylidene)benzenesulfonamide (13)

${ }^{1} \mathbf{H}$ NMR $\left(400 \mathrm{MHz}, \mathrm{CDCl}_{3}\right) \delta 8.74(\mathrm{~d}, 1 \mathrm{H}, J=9.5), 7.89(\mathrm{~d}, 2 \mathrm{H}, J=8.9), 7.54-7.40(\mathrm{~m}$, $6 \mathrm{H}), 7.00-6.93(\mathrm{~m}, 3 \mathrm{H}),, 3.85(\mathrm{~s}, 3 \mathrm{H})$

${ }^{13}$ C NMR $\left(100 \mathrm{MHz}, \mathrm{CDCl}_{3}\right) \delta 170.5,163.7,153.8,134.2,131.7,130.2,129.7,129.3$, $128.7,124.7,114.5,55.8$

IR (thin film) $v$ 3054, 2987, 2305, 1581, 1499, 1421, 1266, 1153, 1092, 896, 857, 807, $743,705 \mathrm{~cm}^{-1}$ 
ESI-MS: $302.1 \quad(\mathrm{M}+\mathrm{H})$

MP: $125-190^{\circ} \mathrm{C}$

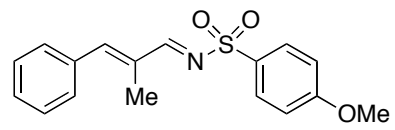

(E)-4-methoxy- $N$-((E)-2-methyl-3-phenylallylidene)benzenesulfonamide (15)

${ }^{1} \mathrm{H}$ NMR $\left(400 \mathrm{MHz}, \mathrm{CDCl}_{3}\right) \delta 8.70(\mathrm{~d}, 1 \mathrm{H}, J=0.5), 7.93(\mathrm{~d}, 2 \mathrm{H}, J=8.9), 7.51-7.40(\mathrm{~m}$, $5 \mathrm{H}), 7.27(\mathrm{~m}, 1 \mathrm{H}), 7.01(\mathrm{~d}, 2 \mathrm{H}, J=8.9), 3.85(\mathrm{~s}, 3 \mathrm{H}), 2.17(\mathrm{~d}, 3 \mathrm{H}, J=3.2)$

${ }^{13}$ C NMR $\left(100 \mathrm{MHz}, \mathrm{CDCl}_{3}\right) \delta 174.3,163.7,151.2,135.3,135.1,130.5,130.3,130.2$, $130.1,128.9,114.5,55.9,13.1$

IR (thin film) v 3054, 2987, 2305, 1566, 1499, 1321, 1265, 1153, 1092, 833, 807, 739, $700 \mathrm{~cm}^{-1}$

ESI-MS: $\quad 316.1 \quad(\mathrm{M}+\mathrm{H})$

MP: $88-90^{\circ} \mathrm{C}$

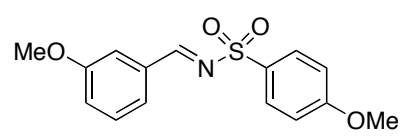

(E)-N-(3-methoxybenzylidene)-4-methoxybenzenesulfonamide (9)

${ }^{1} \mathbf{H}$ NMR $\left(400 \mathrm{MHz}, \mathrm{CDCl}_{3}\right) \delta 8.95(\mathrm{~s}, 1 \mathrm{H}),, 7.92(\mathrm{~d}, 2 \mathrm{H}, J=8.7), 7.46-7.36(\mathrm{~m}, 3 \mathrm{H})$, $7.15-7.13(\mathrm{~m}, 1 \mathrm{H}), 7.00(\mathrm{~d}, 2 \mathrm{H}, J=8.7), 3.86(\mathrm{~s}, 3 \mathrm{H}), 3.82(\mathrm{~s}, 3 \mathrm{H})$

${ }^{13} \mathrm{C}$ NMR $\left(100 \mathrm{MHz}, \mathrm{CDCl}_{3}\right) \delta 169.8,163.9,160.2,133.8,130.5,130.2,129.5,125.4$, $122.2,114.6,55.8,55.7$

IR (thin film) $v 3054,2987,2305,1597,1577,1266,1155,896,811,736 \mathrm{~cm}^{-1}$

ESI-MS: $306.1 \quad(\mathrm{M}+\mathrm{H})$

MP: $198-201^{\circ} \mathrm{C}$

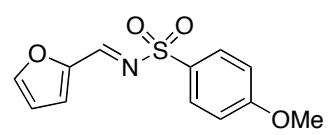

(E)-N-(furan-2-ylmethylene)-4-methoxybenzenesulfonamide (11)

Imine $\mathbf{1 1}$ was prepared according to the procedure of Harris J. M. et $\mathrm{al}^{3}$.

A sample of 2-furaldehyde ( $0.24 \mathrm{~g}, 2.5 \mathrm{mmol}, 1.3$ equiv), 4-methoxybenzenesulphonamide ( $0.37 \mathrm{~g}, 2.0 \mathrm{mmol}, 1.0$ equiv), $5 \mathrm{ml}$ toluene, and p-toluenesulfonic acid (7.6 $\mathrm{mg}, 0.040 \mathrm{mmol}, 2.0 \mathrm{~mol} \%$ ) was placed in a round-bottom flask with a Dean Stark trap and heated at reflux for $10 \mathrm{~h}$. The reaction turned a deep brown color. After $10 \mathrm{~h}$, charcoal was added to the hot solution and the mixture was stirred for $1 \mathrm{~h}$ and filtered.

(3) Harris, J. M.; Padwa, A., J. Org. Chem. 2003, 68, 4371-4381 
The solvent was removed and the product crystallized from benzene to give the desired product (424 mg, $80 \%$ yield) as brown crystals.

${ }^{1}$ H NMR (400 MHz, $\left.\mathrm{CDCl}_{3}\right) \delta 8.79(\mathrm{~s}, 1 \mathrm{H}),, 7.91(\mathrm{dd}, 2 \mathrm{H}, J=6.9,2.0), 7.74(\mathrm{~d}, 1 \mathrm{H}, J=$ $0.8), 7.33$ (d, $1 \mathrm{H}, J=3.7), 6.99(\mathrm{dd}, 2 \mathrm{H}, J=6.9,2.0), 6.64(\mathrm{dd}, 1 \mathrm{H}, J=3.7,1.7), 3.86$ (s, $3 \mathrm{H})$

${ }^{13}$ C NMR $\left(100 \mathrm{MHz}, \mathrm{CDCl}_{3}\right) \delta 163.9,155.4,149.8,149.2,130.5,129.7,124.7,114.6$, $113.9,55.9$

IR (thin film) $v$ 3054, 2985, 2305, 1598, 1546, 1265, 1160, 896, 821, 738, $705 \mathrm{~cm}^{-1}$ ESI-MS: $266.0 \quad(\mathrm{M}+\mathrm{H})$

MP: $97-99^{\circ} \mathrm{C}$

\section{NOE Experiment of Product 10}

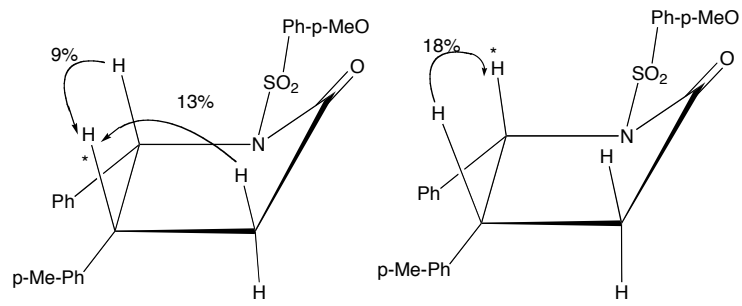




\section{NMR Studies of Catalyst Inhibition}

In a NMR tube was dissolved the enal (1.0 equiv), imine (1.0 equiv) and IMes- $\mathrm{Cl}(0.5$ equiv) in $\mathrm{CD}_{3} \mathrm{OD}$, and the ${ }^{1} \mathrm{H}-\mathrm{NMR}$ spectrum was measured. After addition of 0.5 equiv DBU to the mixture the reaction was followed by the ${ }^{1} \mathrm{H}-\mathrm{NMR}$ was.

1) See Page 15 for ${ }^{1} \mathrm{H}-\mathrm{NMR}$ spectrum.

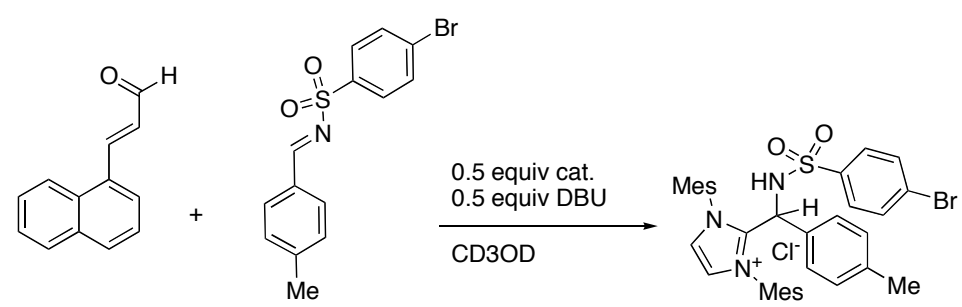

2) See Page 16 for ${ }^{1} \mathrm{H}-\mathrm{NMR}$ spectrum.

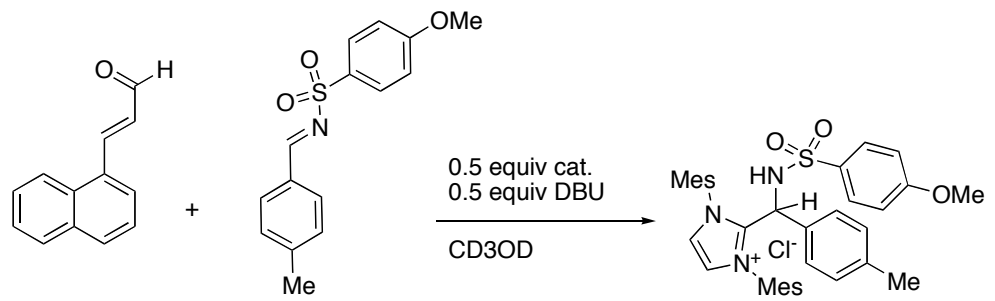



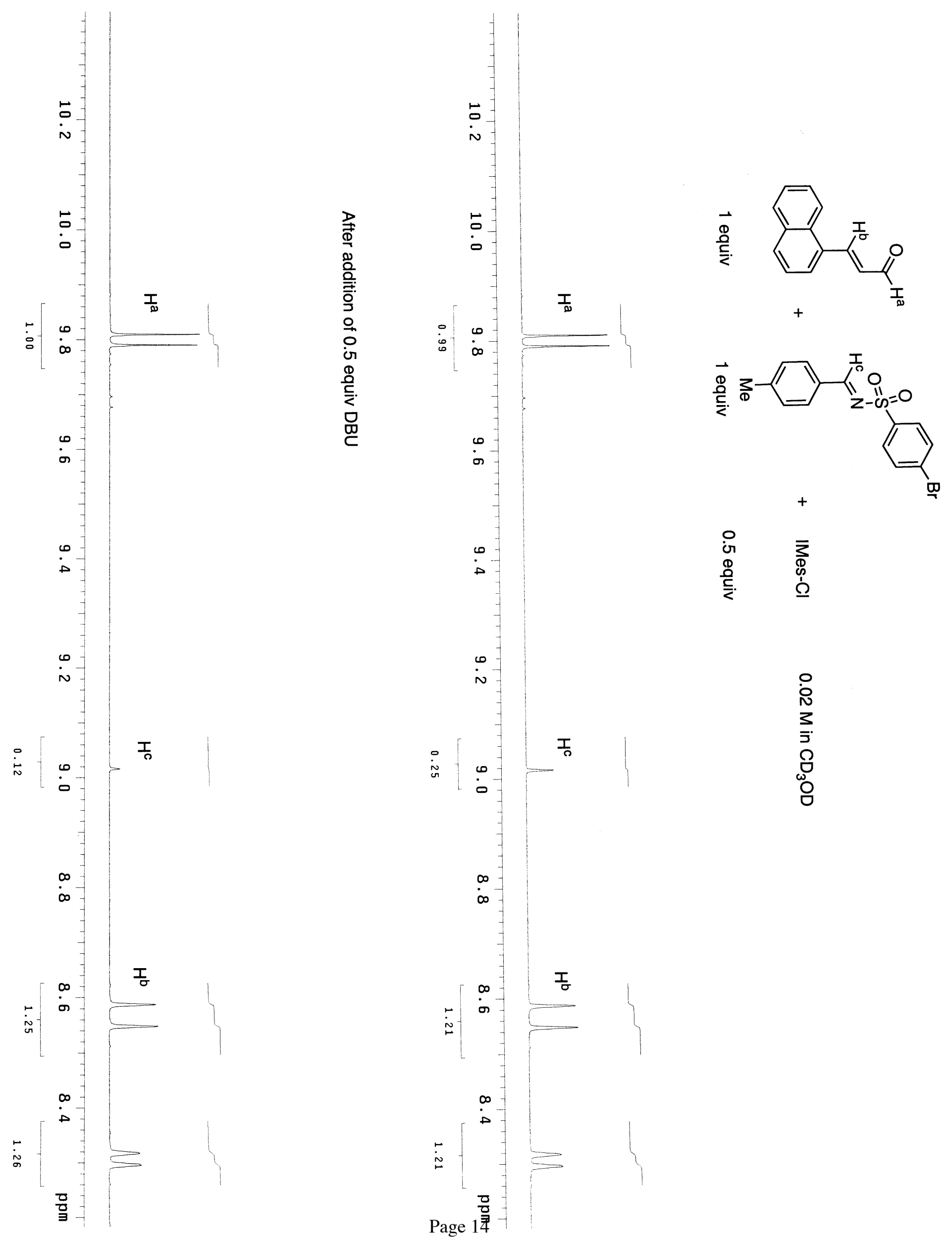

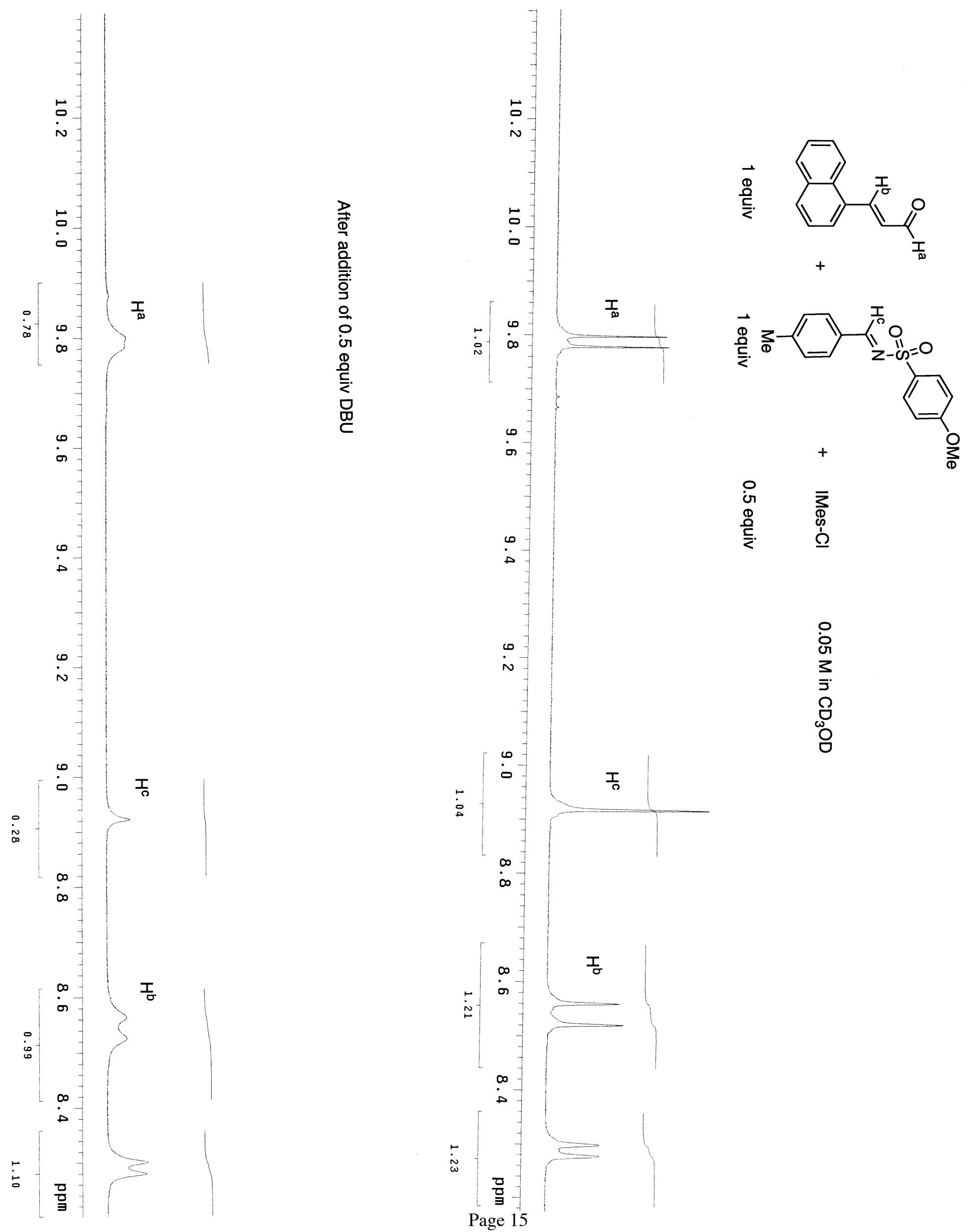


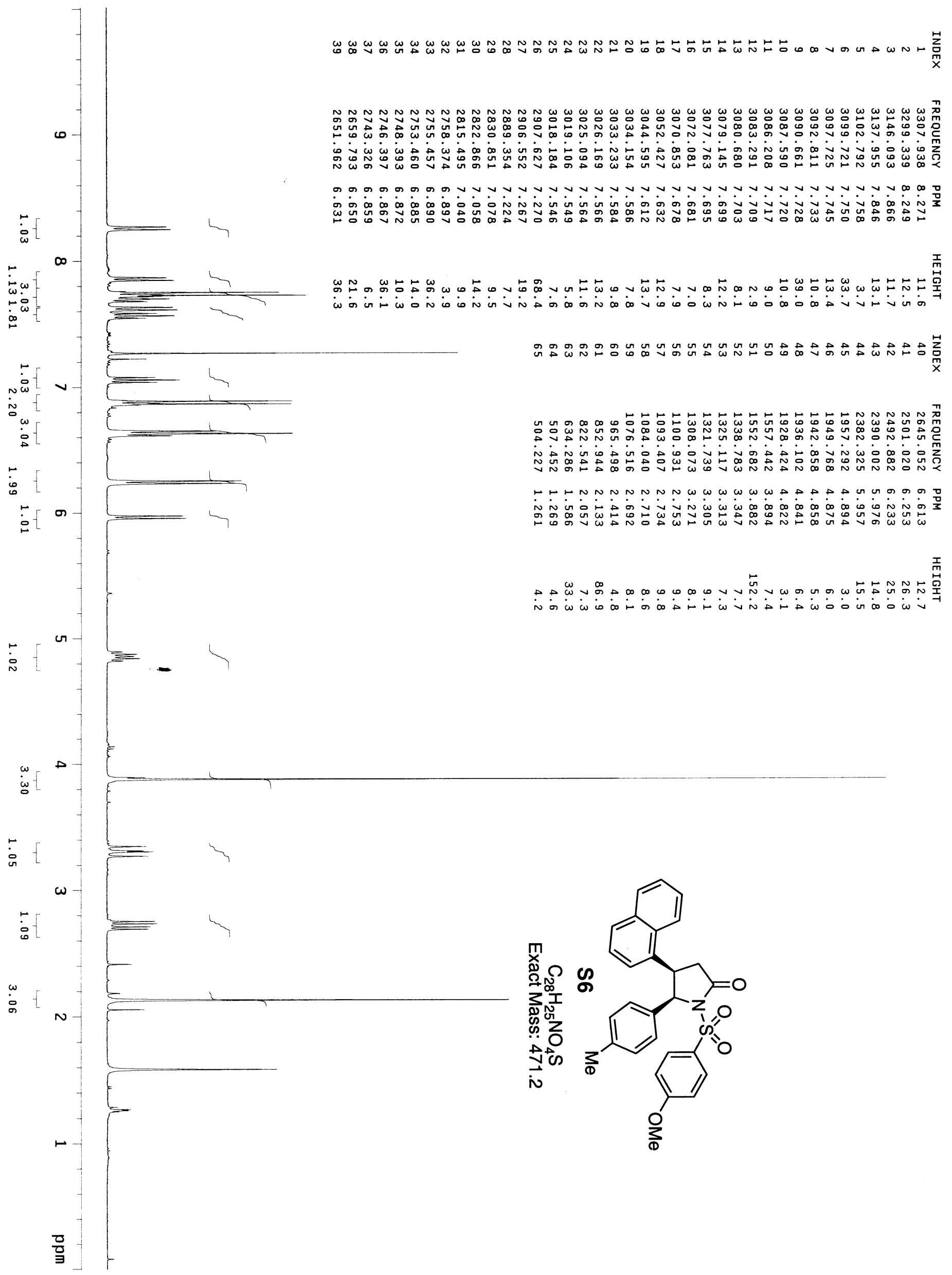




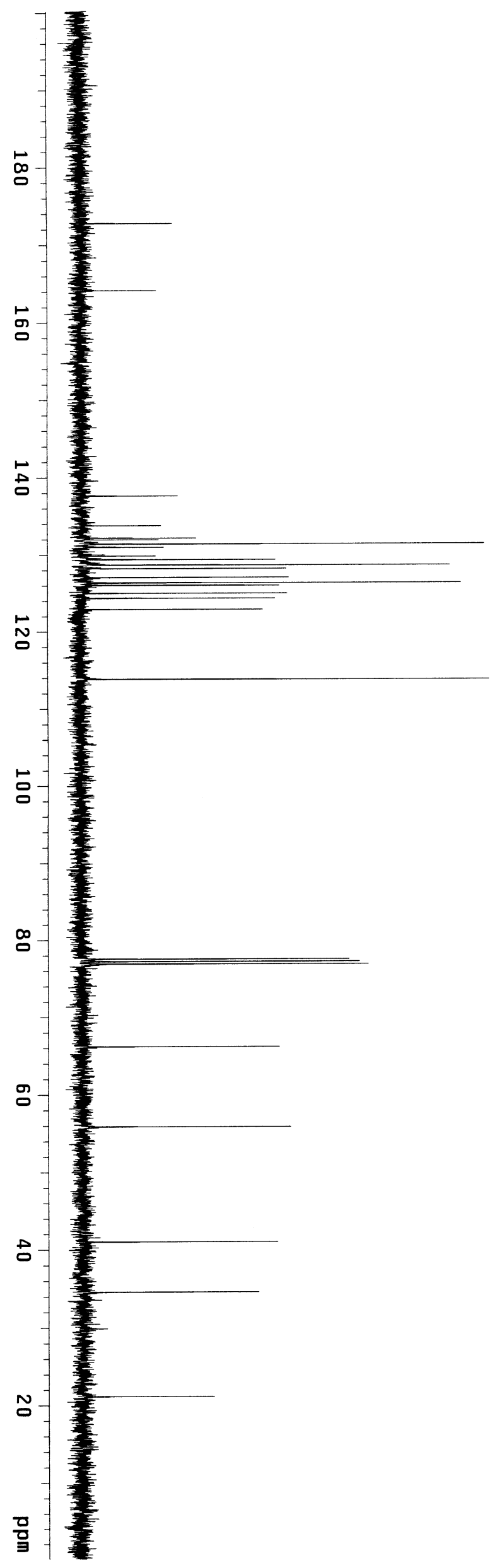

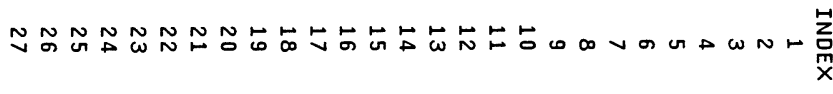

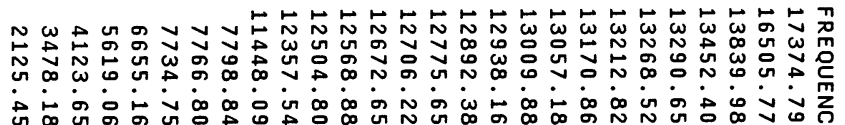

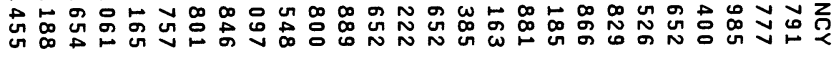

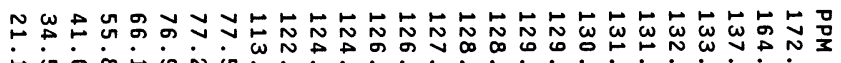

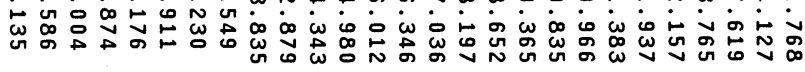

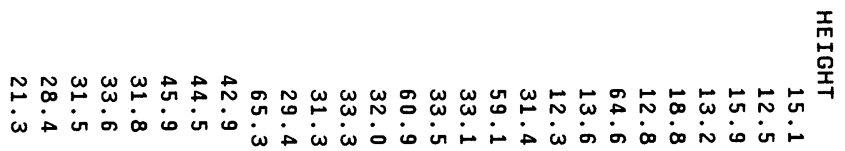

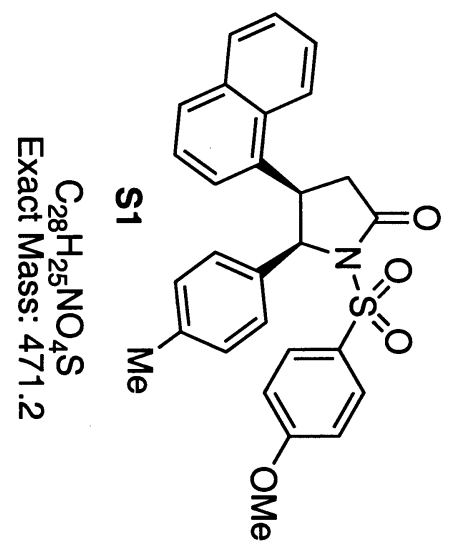




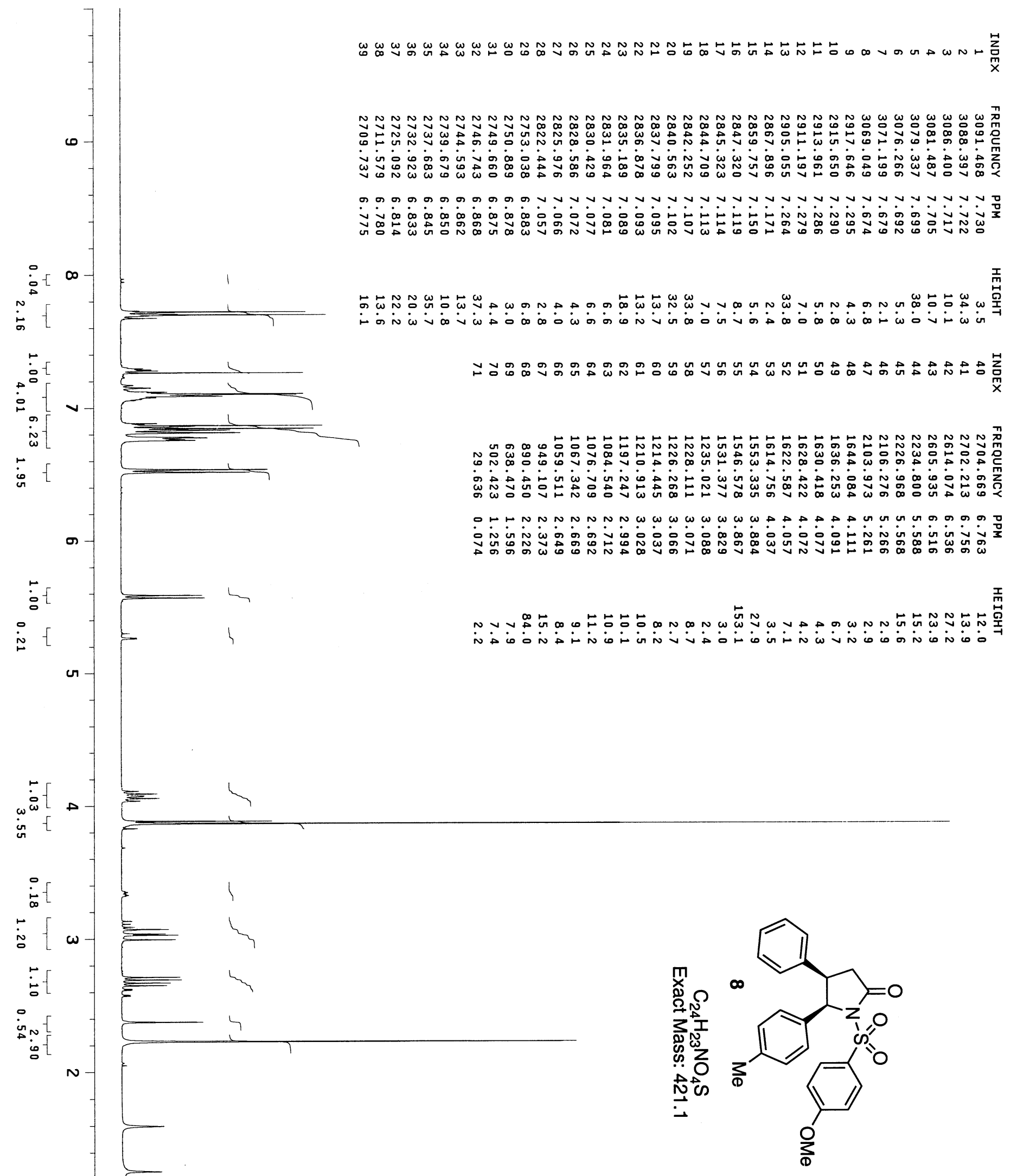

Page 18 


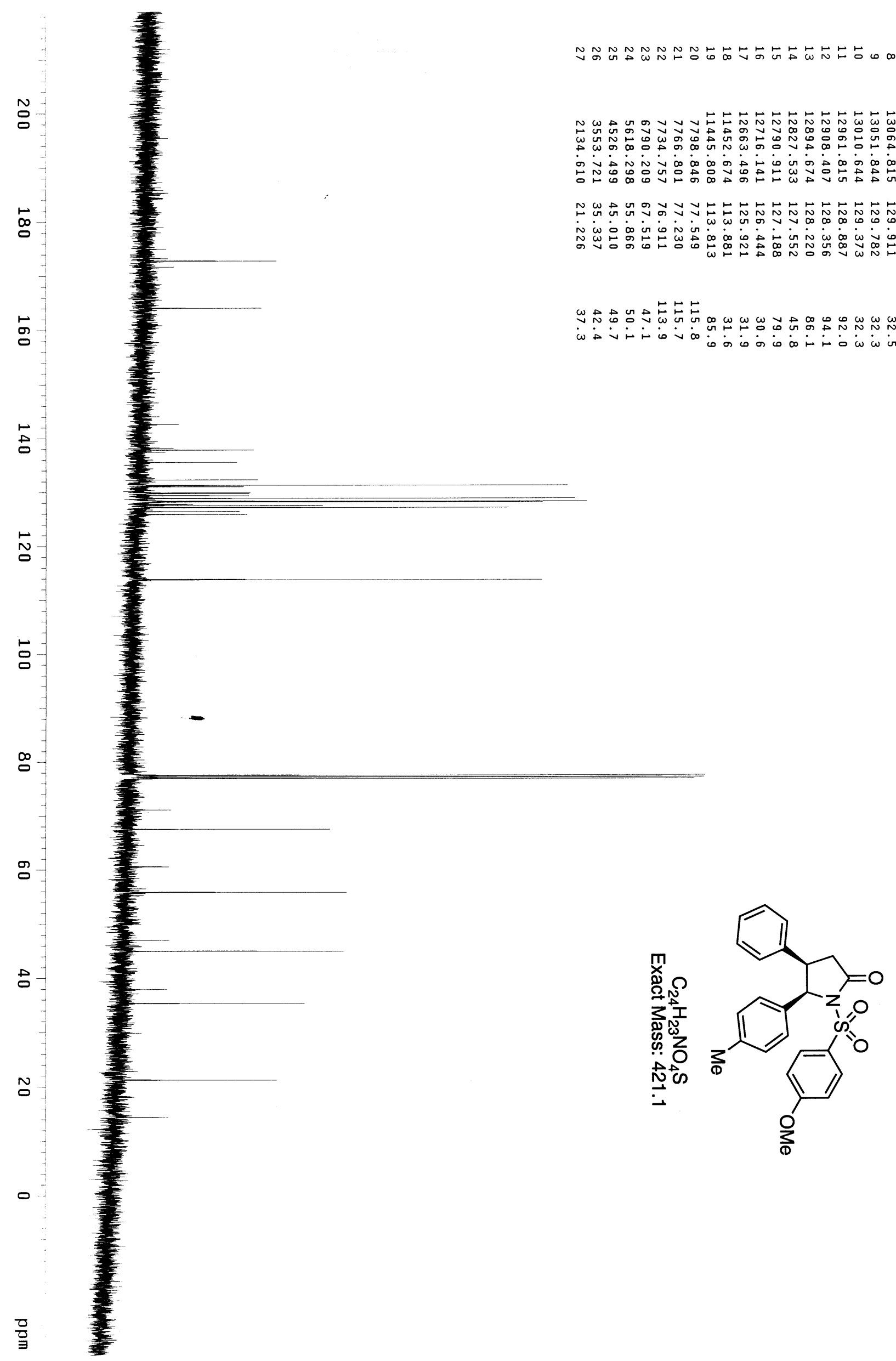




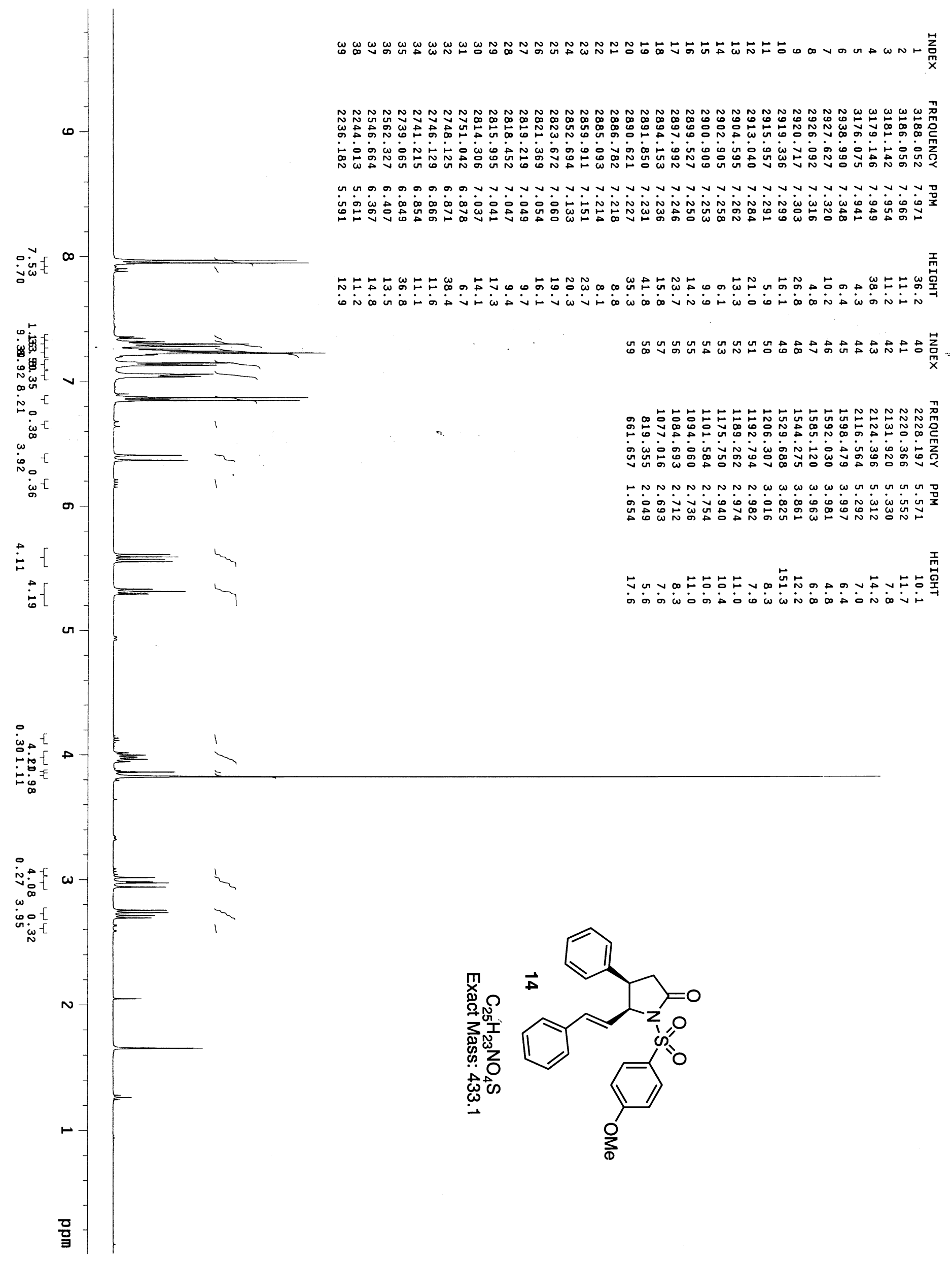



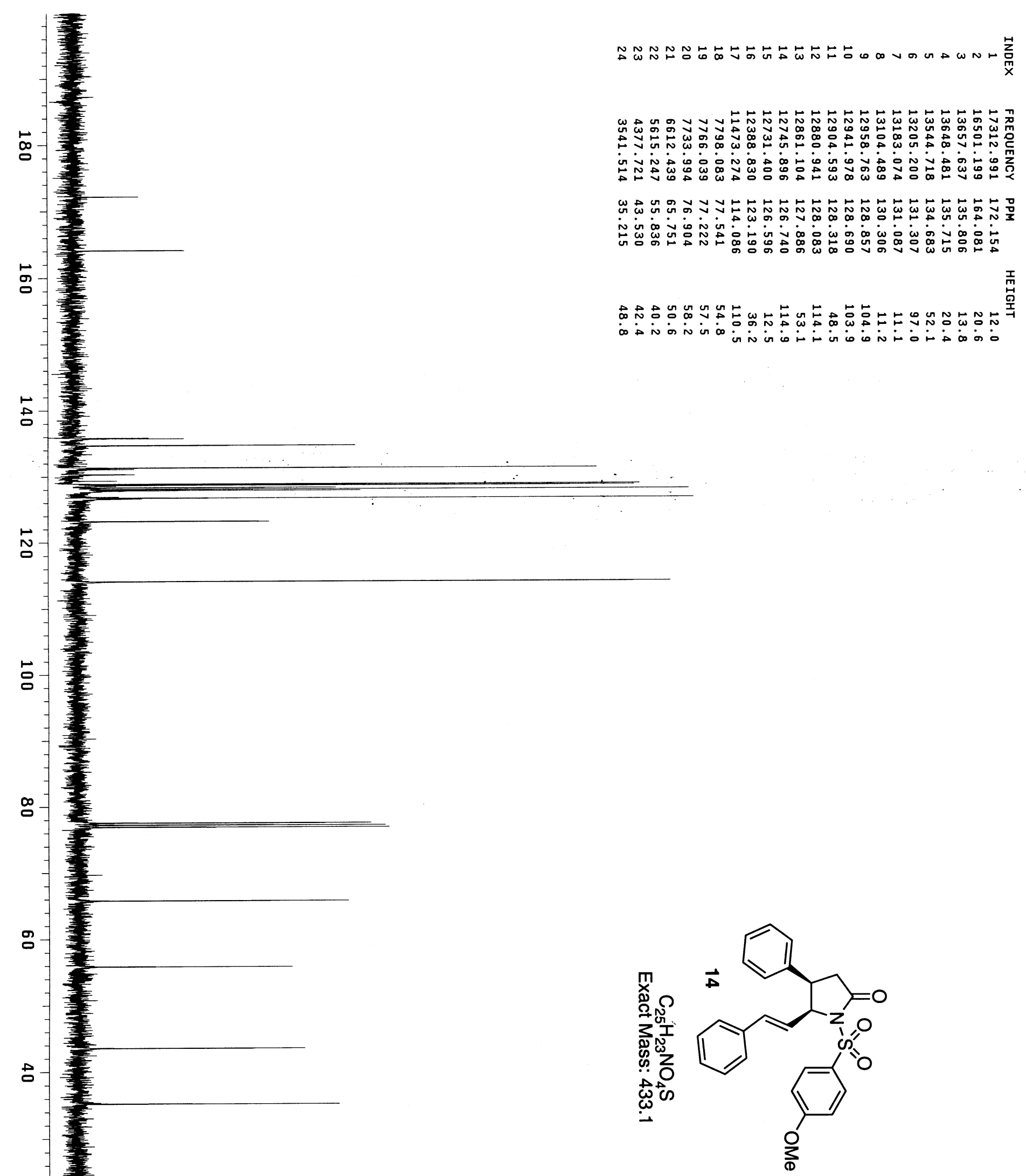